\title{
Experimental researches of determination of degree of interaction of a working organ with the object of treatment in plant growing
}

\author{
Anna I. Belousova ${ }^{1, *}$, and Arkady V. Moiseev ${ }^{1}$ \\ ${ }^{1}$ Federal State Budgetary Educational Institution of Higher Education "Kuban State Agrarian Univer- \\ sity named after I.T. Trubilin", Krasnodar, Russian Federation
}

\begin{abstract}
The article is devoted to the main soil treatment with a combined plow. The article has a research character, which is expressed in the fact that the analysis of methods and means of mechanization for the main tillage with the turnover of a layer, as well as excerpts from the works of leading scientists on the problem, there were shown the types of patents that provided the optimal soil treatment, their shortcomings and solutions to these problems. There were determined the main trend of development of soil-processing combined machines, improvement of constructions of arable units to improve the quality of their work. Also, the form of the disk was justified, namely, there was shown the causal relationship of the presence of cuts in the disk of the tillage tool in comparison with the solid disk. There were described the principle and process of the developed combined plow. As a result of the work done, there was obtained the design of a combined plow with additional working bodies which consist of disks installed by batteries behind each body of the plow. The conclusions set out the main results achieved so far.
\end{abstract}

The mechanical influence of the plow on the soil is accompanied by resistance, which value depends on many factors. Many researchers, having determined the relationship between the physical and mechanical properties of the soil and its quality processing agents, give different forms of calculations using different formulas. They are aimed at determining the traction resistance when plowing with various soil processing tools. Also, leading scientists identify several factors that affect the quality of plowing and the energy process of tillage:

- physical and mechanical (hardness, stickiness, humidity, porosity, etc.)

- technical (type of soil processing unit design, condition, adjustment, etc.)

- technological (way of processing, depth of plowing, speed of processing, etc.)

It is also generally accepted that the traction resistance of soil cultivating unit consists of useful and harmful resistance of its working bodies. The value of useful resistances depends on the size and geometric shapes of the used plows and plow bodies, the depth of processing, as well as on the friction properties of the materials used in the manufacture of working bodies. Harmful resistances depend on the friction force of field boards on the vertical wall of the furrow, on the degree of depreciation of the blades of the working bod-

${ }^{*}$ Corresponding author: anna-lepshina93@mail.ru 
ies, on the load on the support wheels of the propulsive agent and trolleys of working bodies as well as on the resistance to their rolling [1].

In the works of many scientists there were determined the dependences and there were revealed the patterns of influence of individual factors on the energy intensity of the soil treatment process by various tools. Taking into account these circumstances, it is possible to make and present a classification of ways to reduce energy costs for soil treatment.

We developed the plow, namely, the additional working body, has a number of structural features in Figure1. The location of additional working bodies on the frame of the plow does not lead to a complication of the design. The use of an additional working body in the form of an additional flat-cutting working body located on the frame of the plow leads to an increase in qualitative and quantitative indicators of the arable unit.

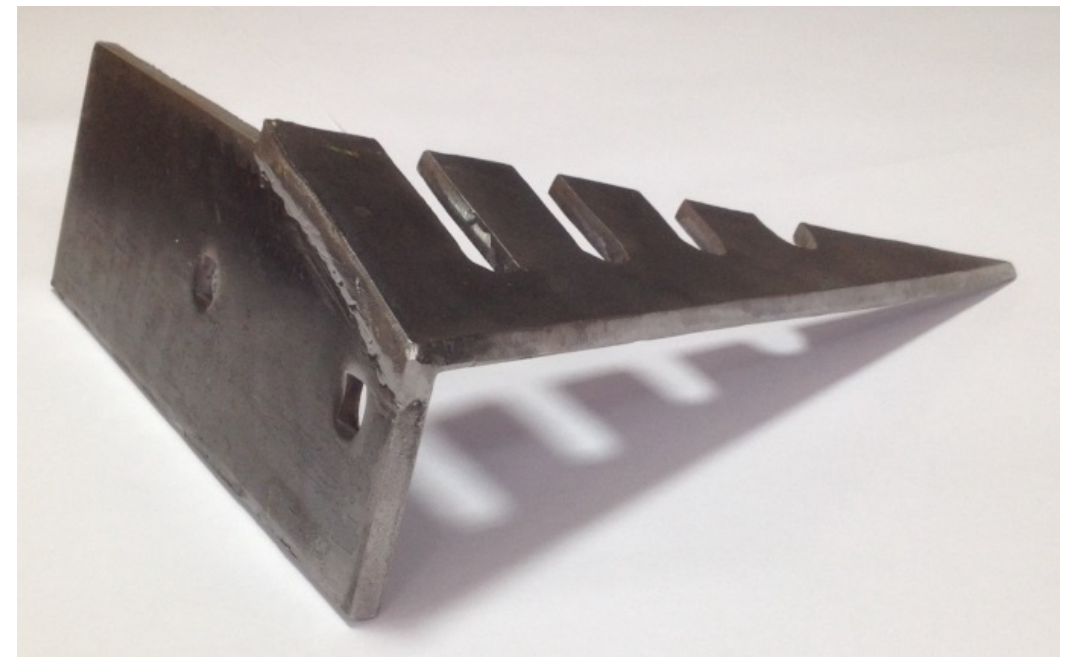

Fig. 1. Developed additional working organ.

The technical result of the proposed design is a flat-cutting working body with additional angle of attack, with reasonable areas of cuts, providing a reduction in traction resistance and soil treatment quality-completeness of soil cutting - and improvement of the quality of work across the width of the soil tillage to complete wear of the flat-cutting working body.

The above technical result is achieved by the fact that the cuts of the flat-cutting working body are located in the area of the working body, taking into account the wear of the part during its operation, and the maximum permissible total area of the cuts, which retains the required structural strength, provides the higher stability of the arable unit. With this technical solution, the quantitative and qualitative indicators of processing of overdried soils are improved, and under humid or overwetted soils, the treatment occurs without sticking and as a consequence, the reduction of the traction resistance of the arable unit.

The normal component of this voltage causes forces of sliding friction tangential-valued component that has a shearing stress due to friction. The essence of design can be explained as follows in detail: when the processing of the soil tillage by working bodies arise the boundary surfaces between the soil and the surface of the working bodies, and more specifically, between soil and steel, namely, between adjacent to each other soil bodies between a thin layer of soil adhering to the working body and the formation of soil, the surface of the working body. In general, such relative displacements cause stresses acting across the surface of the separation of the two bodies-the soil layer - on the material of the flat-cutting working body, i.e., about steel and soil layer on the soil, stuck to the surface of the working 
body. The normal component of this stress causes sliding friction forces, the tangential component of which is the shear stress caused by friction.

According to A. Coolen and K. H. Cooipers the dependence of soil characteristics and materials from conditions of soil sticking on the disk can express the expression of normal

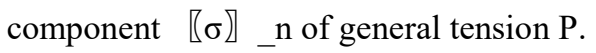

$$
\sigma_{n}<\frac{a-c}{\operatorname{tg} \varphi-\mu^{\prime}}
$$

where a - adhesion;

$\mathrm{c}$ - cohesion;

$\varphi$ - angle of interior friction;

$\mu^{\prime}$ - coefficient of soil friction on material of flat-cutting working body (steel).

Therefore, growth $\sigma_{n}$ will promote the advancement of soil without sticking. So, in this connection, it is reasonable to apply the flat-cutting working body with cuts which increase $\sigma_{n}$, because $\sigma_{n}=\frac{P_{n}}{S}$, where $S$ - general area of soil contact with the working body of the disk, $P_{n}-$ normal component of general tension $P$.

Friction of the soil when it slides on the working surface and its sticking is different phenomena, but they appear themselves simultaneously. Note, however, that if the resistance to sliding friction is not dependent on the area of their conformity to each other, i.e. $\mathrm{F}=f \mathrm{~N}$, the resistance to sliding from adhesion depends on the area of their contact S. They cause the total resistance to sliding $\mathrm{T}$ which is characterized by the following equation [4].

$$
T=F+T_{n p}=f N+p_{0} S+p N S
$$

where F - resistance of sliding of soil to steel (to surface of flat-cutting working body); Тпр-resistance to sliding from sticking;

po - coefficient of tangent forces of specific sticking under absence of normal pressure;

$f$ - coefficient of friction $/$ sliding;

$\mathrm{S}$ - area of contact of soil with area of flat-cutting working body;

$\mathrm{p}$ - coefficient of tangent forces of specific sticking caused by normal pressure.

Analysis of the expression (2) also shows that the total resistance $\mathrm{T}$ can be reduced by reducing the area of possible adhesion. Therefore, from the point of view of reducing the traction resistance, it is necessary to increase the surface area of the cuts of the flat-cutting working body and at the same time the limit of increasing the cut-out area can only be the preservation of the required surface strength of the working body. The location of the slits of the flat-cutting working body with the recommended geometric parameters will provide a reduction in the traction resistance of the arable unit, increases its permeability during soil processing, reduces the traction resistance, ensures the stable movement of the soil layer and the cutting of weeds along the entire width of the processing, that is, ensures the achievement of technical result.

We propose to install a battery of flat discs on individual beams directly behind each body of the plow, namely behind the mould board, so that the battery melted the wrapped layer of soil with the frame of the plow and thereby giving it a more fine-grained structure.

A flat disk is usually used as a disk knife for cutting off the formation in the vertical plane. The forces of resistance of the soil to crumple the blade and the force of friction of the soil on its side surfaces influence on the knife. Experiments (conducted by 
Cand.Tech.Sc., Ph. D. Mamatov) showed that the influence of friction forces is relatively small. Since the disk knife is a symmetrical working body, the action of the elementary forces of soil resistance can be reduced to one equally active $\mathrm{AB}$ figure 4 and passing through the axis of rotation. The $\mathrm{R}_{\mathrm{x}}$ component of this force is the traction resistance of the knife and creates a moment that promotes the rotation of the disk. Another component of $\mathrm{R}_{\mathrm{z}}$ seeks to push the knife out of the soil and creates a moment that prevents the rotation of the disk. If you change the resistivity of the soil from 40 to $80 \mathrm{kPa}$ the power $\mathrm{R}_{\mathrm{x}}$ of standard knife plow of general purpose varies from 0.7 to $2.2 \mathrm{kN}$. At the same time $\mathrm{R}_{\mathrm{z}} \approx 1,2 \mathrm{R}_{\mathrm{x}}$.

Therefore, in our case, in order the knife to cut the wrapped layer of soil with a plow, but not roll over the ridges, it is necessary that the angle of binding $\delta$ is less than $\varphi 1+\varphi 2$, where $\varphi 1$ is the angle of friction of the soil to the blade, and $\varphi 2$ is the angle of friction of the disk to the soil. If the knife disk is clogged from the density of the soil, it is necessary to reduce the depth of its motion or to put a knife of a larger diameter. (Klenin N.I., Sakun V. A. Agricultural and land reclamation machines: Elements of the theory of working processes, calculation of adjusting parameters of work. - 2nd ed., added. - M.: Kolos, 1980. P.90).

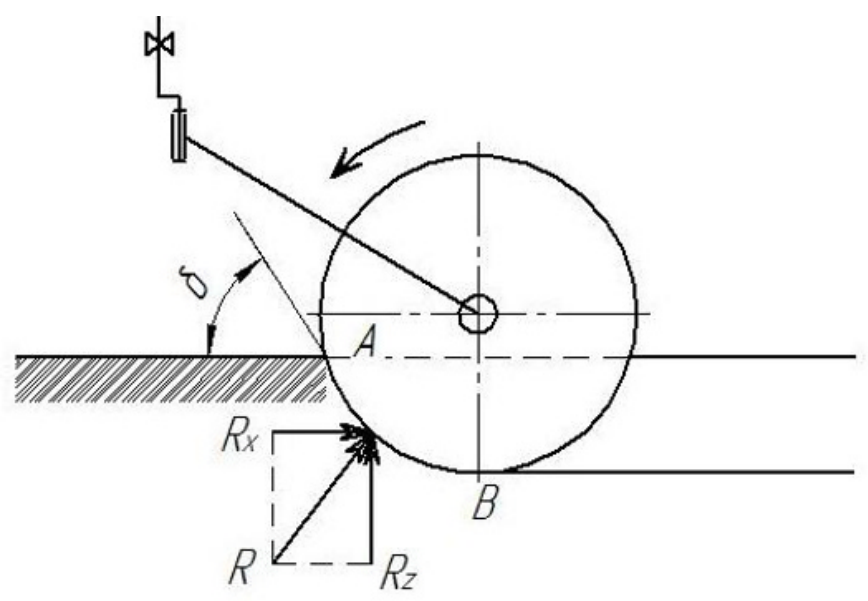

Fig. 2. Force characteristics of flat knife

In general, in such relative displacements there are stresses acting across the surface of the separation of the two bodies - the soil layer on the material of the flat disk, i.e. on the steel and the soil layer on the soil adhering to the surface of the flat disk. The normal component of this stress causes sliding friction forces, the tangent component of which is the shear stress caused by friction. Therefore, from the point of view of reducing the traction resistance, it is necessary to increase the surface area of the flat disk and at the same time the limit of increasing the area of the cut-out can only be the preservation of the required strength of the disk in Figure 3. 


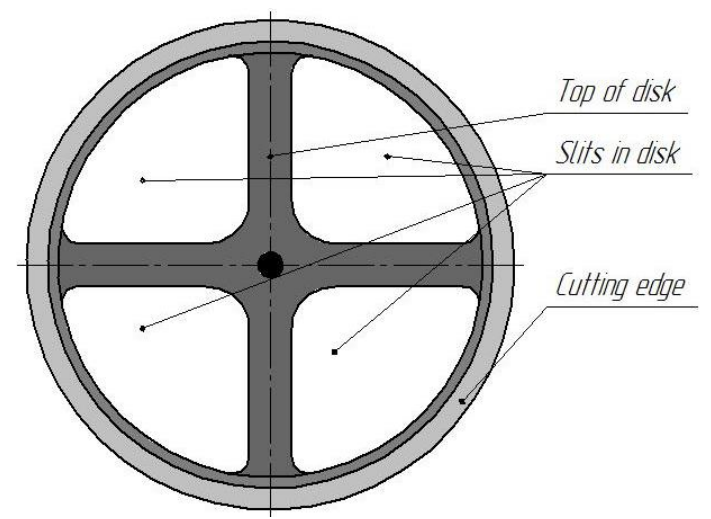

Fig. 3. Flat disk with slits.

In the result of the work of the proposed design is the reduction of steel intensity in comparison with a similar combined tillage tools, traction resistance and increase of soil processing quality by reducing the traction resistance of the plow and the increase of quantitative and qualitative indicators of work of the arable unit with a new working bodies.

The specified result is achieved in that the flat disks assembled in a battery and are mounted on jointed individual beams for each plow, wrapped layer of soil occurs under battery of disks which destroy it into smaller fractions, thereby reducing the further number of passes of heavy units on the field, thereby reducing the production costs of agricultural products.

The essence of the described design is that we install a battery of disks 9 on the individual beams 7, 8 directly behind each body of the plow. The working body is made in the form of a solid disk with cuts in the middle part to reduce the metal content and weight of the structure. The beam 7.8 has a jointed construction 10 that allows to transfer the battery of disks into transport position without their disconnecting from the plow.

Machine for soil processing works as follows: plow entering the soil with bodies consisting of frames 2 plow 3 bearing plate 4 field board 5 mould board 6 cuts the soil layer and taking it by the mould board 6 of the body and returns it in the bottom of the furrow, mounted on the frame of the plow 1 on the individual beams 7.8 battery of disks 9 entering the wrapped soil layer with their cutting edges, crumbles it into smaller fractional components, and thereby giving the surface of the field-aligned structure, closing moisture in it and embedded weeds at the bottom of the furrow lost the link with the external environment, which leads to their natural death. 

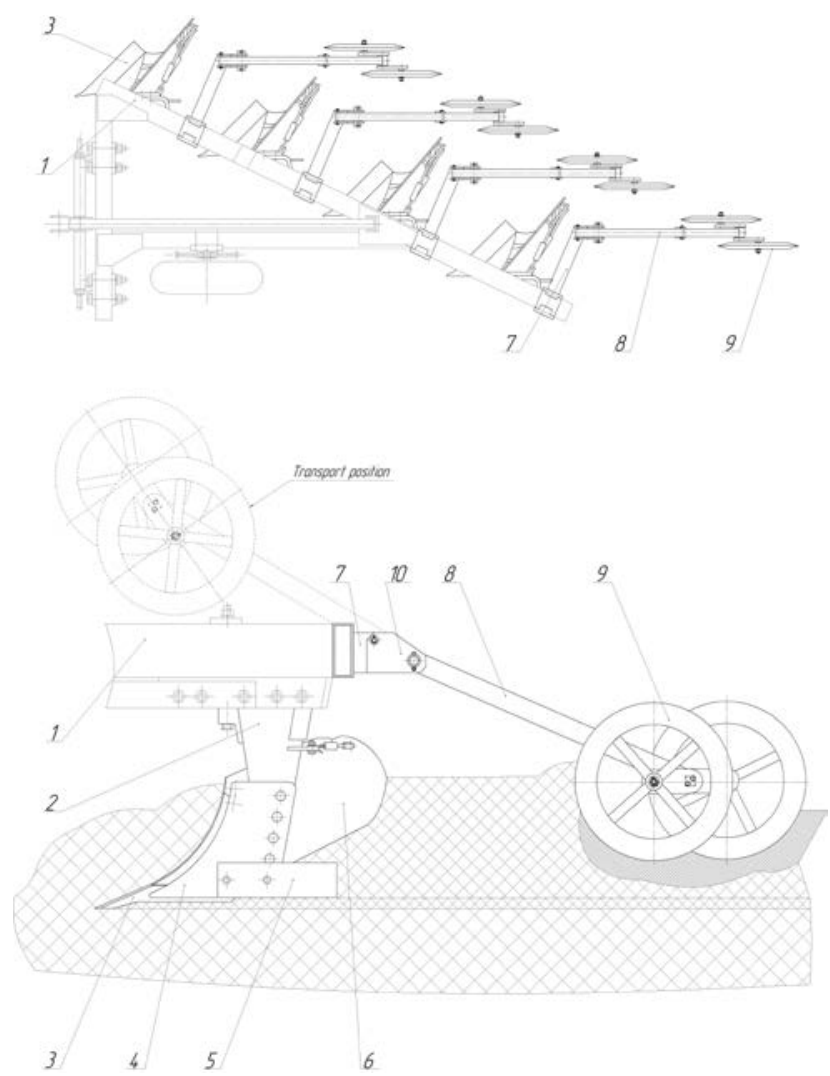

1- Rama; 2 - Rack; 3 - Shaped; 4 - Shoe; 5 - field board; 6 - Blade; 7, 8 - Gyradil; 9 - Disks; 10 - Hinge.

Fig. 4. Proposed construction of combined plow

In the conclusion of the work you can do the following: development of additional working bodies for the improvement of the process of main processing of the soil, gives the opportunity to improve qualitative and quantitative parameters of the process operation. These qualities of soil treatment were confirmed during field experiments, and the main structural elements were patented. The use of additional bodies of the present design allows to produce the main soil preparation with less costs of fuel and, as a result, to achieve the better quality indicators for the cultivation of agricultural products.

\section{References}

1. Trubilin E.I., Belousov S.V., Lepshina A.I., Results of experimental studies determining the degree of traction resistance of the plowshoe in the treatment of heavy soils, The Political Network Electronic Scientific Journal of the Kuban State Agrarian University, No. 103, 673-686 (2014)

2. Trubilin E.I., Belousov S.V., Lepshina A.I., Economic efficiency of the dump cultivation of soil developed by a combined plowshoe, The political network electronic scientific journal of the Kuban State Agrarian University, No. 103, 654-672. (2014) 
3. Trubilin E.I., Belousov S.V., Lepshina A.I., The main soil cultivation with the formation turnover in modern working conditions and devices for its implementation, The political network electronic scientific journal of the Kuban State Agrarian University, No. 104, 1902-1922 (2014)

4. Belousov S.V., Communication of science and technology in the field of development of machines for the basic tillage of the soil with the circulation of the bed, The political network electronic scientific journal of the Kuban State Agrarian University, No. 109, 468-486 (2015)

5. Belousov S.V. Modern technologies of soil cultivation, Scientific provision of the agro industrial complex, 2012, 3-4.

6. Parkhomenko GG, Bozhko I.V., Semenikhina Y.A., Pantyukhov I.V., Drozdov S.V., Gromakov A.V., Kambulov S.I., Belousov S.V., Perfection of working organs for tillage, State and prospects of the development of agricultural machinery, Collection of articles of the 9th international scientific and practical conference within the framework of the 19th international agro-industrial exhibition "Interagro-mash-2016". -2016, 27-30.

7. Belousov S.V., Trubilin E.I., Development of the construction of a plow for the treatment of heavy soils, Science of the Kuban, No 1, 37-40 (2013)

8. Belousov S.V., Patent search for structures that provide soil treatment with the turnover of the reservoir. Search method. The proposed technical solution, The political network electronic scientific journal of the Kuban State Agrarian University, No. 109, 416-450 (2015)

9. Belousov S.V., Decrease in energy intensity of the process of basic processing of soil with turnover of the bed, Fundamental foundations of modern agrarian technologies and technology. Collection of works of the All-Russian Youth Scientific and Practical Conference. -National Research Tomsk Polytechnic University, 2015, 280-283.

10. Belousov S.V., Role and prospects for the development of small-scale mechanization in the context of co-temporary engineering, Scientific support of the agro-industrial complex. A collection of articles on the materials of the 72 nd Scientific and Practical Conference of pre-applicants on the results of research work for 2016 - 2017.-p. 277278. 